\title{
MICRO-SCALE DIAMOND HEMISPHERICAL RESONATOR GYROSCOPE
}

\author{
P. Taheri-Tehrani ${ }^{l}$, T.-H. Su ${ }^{l}$, A. Heidari ${ }^{l}$, G. Jaramillo ${ }^{l}$, C. Yang ${ }^{2}$, S. Akhbari ${ }^{2}$, H. Najar ${ }^{l}$, S. Nitzan ${ }^{l}$, D. Saito ${ }^{2}$, \\ L. Lin $^{2}$, and D. A. Horsley ${ }^{1}$ \\ ${ }^{1}$ University of California Davis, USA \\ ${ }^{2}$ University of California Berkeley, USA
}

\begin{abstract}
This paper concerns a micro-scale gyroscope based on a $1 \mathrm{~mm}$ diameter diamond hemispherical resonator with embedded polysilicon electrodes. Frequency mismatch and quality factor of the wineglass vibration modes are studied. The device has embedded electrodes used for electrostatic transduction of resonator vibration and for electrostatic mode-matching. The air gap between the resonator and electrodes is small to increase the frequency tuning range for a given supply voltage. The $2 \theta$-mode resonant frequency mismatch can be minimized from $2.4 \%$ to $0.1 \%$ by applying a low $2.5 \mathrm{~V}$ tuning voltage.
\end{abstract}

\section{KEYWORDS}

Gyroscope, Diamond, Hemispherical Resonator, Electrostatic Tuning, Quadrature.

\section{INTRODUCTION}

The principle of the vibratory gyroscope is Coriolis acceleration coupling between two degenerate resonant modes of a vibratory structure. Mechanical structures such as rings, disks, and $4 \theta, \ldots$ modes) which exhibit Coriolis coupling. The Coriolis acceleration amplitude for a given rotation rate, referred to as angular gain, depends on the geometry and mode-shape of the resonator. The main advantage of these structures is that they have a highly symmetric structure, enabling the device to have a low split between the resonant frequencies $(\Delta f / f<1 / Q)$. Appropriately designed electrodes can be used to null the residual frequency and quadrature error through electrostatic tuning.

Here, we present a rate gyroscope based on a $1 \mathrm{~mm}$ hemispherical resonator made of thin-film microcrystalline diamond (MCD). Resonator vibration is excited and sensed using electrostatic transduction using electrodes that also provide electrostatic tuning. First, an overview of the fabrication is presented, then design of the device is discussed. Information on the characteristics of the devices is provided. The devices are tested as gyroscopes and their performance is reported.

\section{DESIGN AND FABRICATION}

The device, shown in Figure 1, is a $1 \mathrm{~mm}$ diameter, $250 \mu \mathrm{m}$ deep hemispherical resonator formed from $1 \mu \mathrm{m}$ thick MCD. The device diameter is four and six times smaller than gyro designs reported in [1-2], respectively. Eight boron doped polysilicon electrodes are embedded in the silicon cavity surrounding the hemispherical resonator with a $1 \mu \mathrm{m}$ capacitive gap. The polysilicon electrodes are isolated with a $1 \mu \mathrm{m}$ silicon nitride $\left(\mathrm{Si}_{3} \mathrm{~N}_{4}\right)$ layer from the <111> silicon substrate. The resonator is anchored to the substrate, enabling dc voltage to be applied through the silicon substrate. A similar structure is presented in [3-4], but no gyroscope performance is reported.

A novel self-aligned process is employed in the fabrication. First, hemispherical molds are formed via a silicon isotropic etch using HNA (HF/nitric/acetic acids) with a $1.2 \mu \mathrm{m} \mathrm{LPCVD} \mathrm{Si}_{3} \mathrm{~N}_{4}$ hard mask. Then, a $1.2 \mu \mathrm{m}$ thick LPCVD $\mathrm{Si}_{3} \mathrm{~N}_{4}$ layer and a $500 \mathrm{~nm}$ boron doped polysilicon layer are conformally deposited inside the silicon molds. The self-aligned electrodes are formed by patterning hemispheres have pairs of degenerate wineglass modes (i.e. $2 \theta, 3 \theta$,
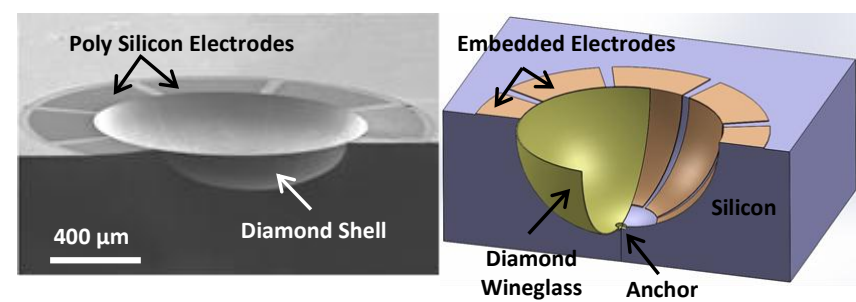

Figure 1: SEM and schematic images of hemispherical resonator gyroscope with embedded electrodes.

the polysilicon layer within the cavities using spray-coating (EVG 101 Spray Coater), lithography and plasma etching. A $1 \mu \mathrm{m} \mathrm{SiO} 2$ layer is deposited as sacrificial layer on the top of the polySi electrodes. Then, the $60 \mu \mathrm{m}$ diameter anchor is patterned, followed by CVD of $1 \mu \mathrm{m}$ boron doped diamond. The diamond on the wafer surface is removed using CMP and $\mathrm{O}_{2}$ plasma etching, after which the resonator is released in HF. The fabrication process flow is illustrated in Figure 2.

The scale factor $\left(S_{\Omega}\right)$ from input rate to output displacement on the sensing axis is:

$$
S_{\Omega}=2 A G \frac{m_{A} Q_{B}}{k_{B}} M(\Delta f) 2 \pi f_{A} q_{A}
$$

where $Q_{B}, m_{B}$, and $k_{B}$ are the quality factor, modal mass, and spring constant of the sense-axis, $q_{A}$ is the displacement of the drive axis, $f_{A}$ is the drive oscillation frequency, $A G$ is the Coriolis coupling (or angular gain), $\Delta f$ is the frequency mismatch between the drive and sense modes and $M(\Delta f)$ represents the normalized magnitude of the sense-axis transfer function evaluated at $f_{A}=f_{B}-\Delta f$,

$$
M(\Delta f)=\left\{Q_{B}^{2}\left[1-\left(1-\Delta f / f_{B}\right)^{2}\right]^{2}+\left(1-\Delta f / f_{B}\right)^{2}\right\}^{-\frac{1}{2}} .
$$

In a mode-matched gyro, $\Delta f=0$ and $M(\Delta f)=1$.

Frequency mismatch and quadrature error arise from imperfections in the resonator that cause the resonator's stiffness matrix to be non-diagonal and to have unequal diagonal entries. These can be corrected using electrostatic tuning [5]. In the current gyro, the tuning electrodes only allow the on-diagonal stiffness components to be adjusted,

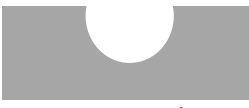

1- HNA etch

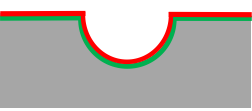

2- SiN \& Poly Deposition

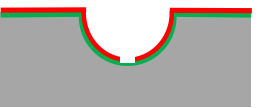

3-PolySi Pattern(Electrod

Silicon $\square \mathrm{SiN}$

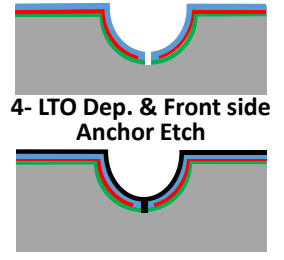

5- Diamond Deposition

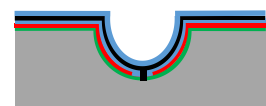

Polysilicon

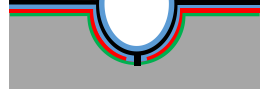

7- CMP, LTO Etching

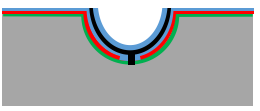

8- Diamond Etching

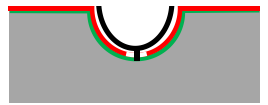

9- HF Release
Figure 2 : Fabrication process of the hemispherical resonator.

Solid-State Sensors, Actuators and Microsystems Workshop Hilton Head Island, South Carolina, June 8-12, 2014 


$$
\left[\begin{array}{cc}
k_{A} & k_{A B} \\
k_{A B} & k_{B}
\end{array}\right]=\left[\begin{array}{ll}
k_{A 0} & k_{A B} \\
k_{A B} & k_{B 0}
\end{array}\right]-\left[\begin{array}{cc}
k_{T A} & 0 \\
0 & k_{T B}
\end{array}\right]
$$

where $k_{A 0}$ and $k_{B 0}$ denote the initial spring constants of the A and $\mathrm{B}$ axes, $k_{T A}, k_{T B}$ denote the electrostatic spring constants, and $k_{A B}$ represents the cross-axis coupling. Cross-axis coupling results in quadrature error; the tuning electrodes used here have an angular extent of $45^{\circ}$ and are therefore unable to change these off-diagonal components to null the quadrature error.

Angular gain $(A G)$ is a measure of Coriolis coupling between the degenerate resonant modes in a gyroscope $(0<A G<1)$. The scale factor is proportional to $A G$, which is solely dependent on the geometry and mode-shape of the resonator. $A G$ can be estimated using the displacement field of the two resonant modes of interest using a FEM model of the structure [6],

$$
\begin{gathered}
\vec{m}_{c}=\sum \overrightarrow{X_{l}} \times \vec{Y}_{l} \cdot m_{i} \\
\overrightarrow{A G}=\frac{\vec{m}_{c}}{m_{e f f}}
\end{gathered}
$$

where the index $i$ refers to each mesh element in the FEM model and $m_{i}$ corresponds to the mass of each element. $\vec{X}_{l}$ and $\vec{Y}_{l}$ correspond to the normalized displacement vectors of the two degenerate modes. $\vec{m}_{c}$ is a three component vector and the absolute value of each component divided by the modal mass of drive resonant mode gives the $A G$ value for each axis. The three components of $\overrightarrow{A G}$ correspond to the angular gain for rotation rate in each axis $\left(\Omega_{x}, \Omega_{y}, \Omega_{z}\right)$.

FEM simulations were conducted to study the effect of the depth-to-radius ratio of the hemispherical resonator on the angular gain. The simulation results, shown in Figure 3, demonstrate the increase in angular gain and reduction in normalized $2 \theta$-mode resonant frequency of the hemispherical resonator as the depth-to-radius ratio increases. However, the depth of devices reported here are limited to $250 \mu \mathrm{m}$ due to the HNA etch used to form the resonator molds.

A numerical calculation was performed based on the analytical mode-shape of the hemispherical resonator [7] to calculate the electrode parameters. The electrode capacitance $(C)$ and displacement sensitivity $d C / d x$ are estimated to be $1.3 \mathrm{pF}$ and 0.6 $\mu \mathrm{F} / \mathrm{m}$, respectively. Modal stiffness and modal mass of the $2 \theta$-mode estimated from FEM simulation are $25 \mathrm{~N} / \mathrm{m}$ and $8 \cdot 10^{-10} \mathrm{~kg}$, respectively. High capacitance and low stiffness result in a wide tuning range: only $1 \mathrm{~V}$ tuning voltage will cause $\sim 1 \mathrm{~N} / \mathrm{m}$

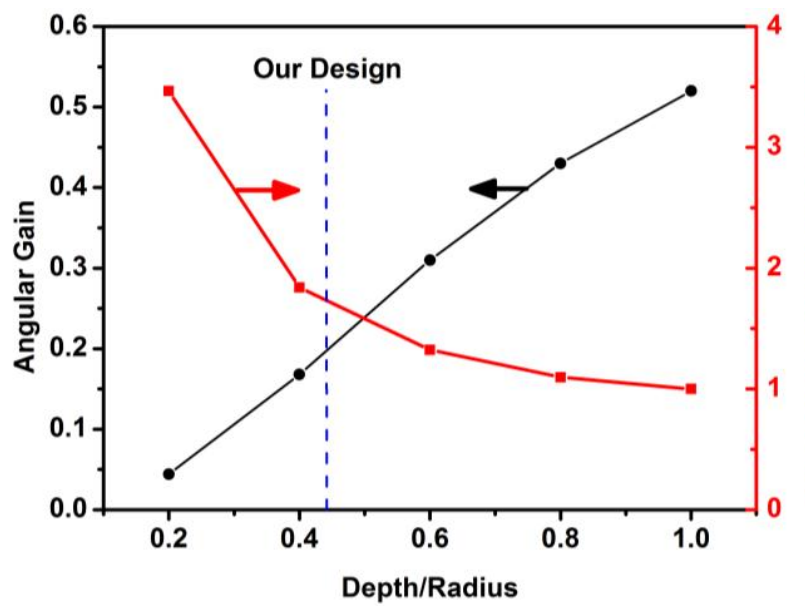

Figure 3 : Angular gain and normalized 20-mode natural frequency versus depth-to-radius ratio. electrostatic softening, corresponding to a $2 \%$ reduction in natural frequency.

\section{RESONATOR CHARACTERIZATION}

The vibration frequency response of several hemispherical resonators was characterized using a Laser Doppler Vibrometer (LDV, Polytec Inc.) both in air and in vacuum. They were mounted vertically onto a shear mode piezoelectric actuator (Noliac A/S) with a bandwidth of $1.7 \mathrm{MHz}$ to excite vibration. The single point LDV laser spot was focused through a 10x microscope objective onto the rim of the shell to measure the radial displacement of the vibrating shell. Measurements of the frequency and Q-factor of the $2 \theta, 3 \theta$ and $4 \theta$ vibration modes are shown in Figure 4. As can be seen in this figure, higher-order modes have higher Q-factor. The $2 \theta$ mode Q-factor of 24,000 was obtained at a pressure $<1 \mathrm{mTorr}$.

The diamond resonator was also mounted in a vacuum probe station (MMR Technologies Inc.) to allow measurement using capacitive excitation and readout via the embedded electrodes. The frequency response of a hemispherical shell in vacuum at $30 \mu$ Torr is shown in Figure 5. The observed resonances at $26.68 \mathrm{kHz}$ and $26.75 \mathrm{kHz}$ correspond to the two $2 \theta$ elliptical vibration modes. The frequency mismatch between these two degenerate resonant modes is $75 \mathrm{~Hz}$, which corresponds to $\Delta f / f$ of $0.28 \%$. The observed Qfactors are approximately 12,100 and 14,800 . The lower $Q$ in observed in this experiment may be due to increased gas damping or from electrical loading from the testing setup.

\section{GYROSCOPE PERFORMANCE}

The hemispherical resonator is surrounded by eight electrodes. Two vibration axes are required for gyroscope operation, one for the drive axis (in this paper, axis A, which corresponds to the mode A resonant frequency) and the other for the sense axis (in this paper, axis B, which corresponds to the mode B resonant frequency). For each axis, two electrodes are used for differential sensing to decrease the feedthrough, one electrode is used for single-ended drive, and one electrode for direct tuning. For rate-sensing experiments, resonator dice were mounted in 28-pin packages using conductive epoxy and wire-bonded to the pads. As shown in Figure 6, the package was mounted on a PCB with differential sense circuitry. A digital lock-in amplifier (Zurich Instruments HF2LI) was used to provide de tuning voltages, closed-loop amplitude control of the drive axis, and demodulate the sense axis vibration.

The resonators have direct tuning electrodes that can be used to compensate for $\Delta f$. However, without quadrature nulling electrodes, devices exhibiting high quadrature cannot be perfectly

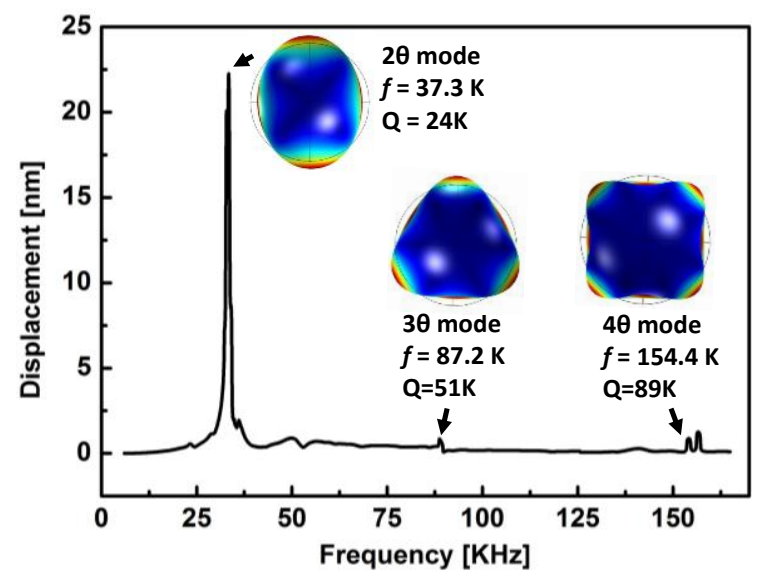

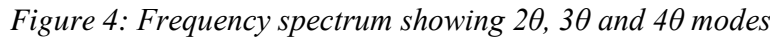
of the wineglass resonator. 


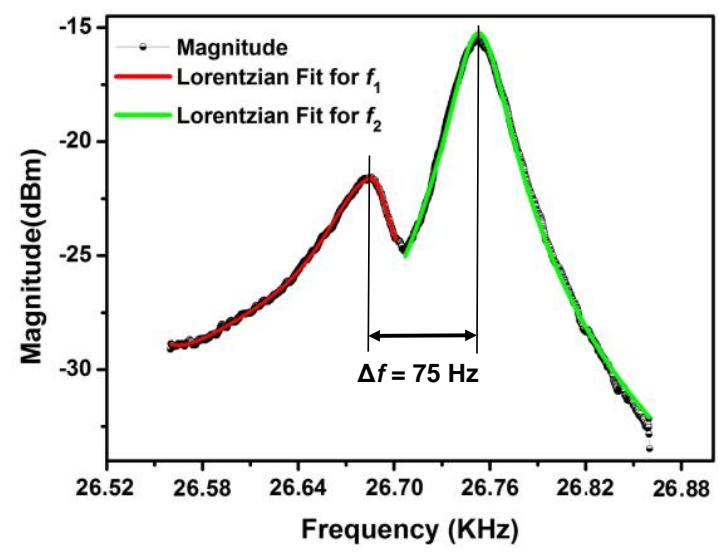

Figure 5: Measured frequency response showing the $2 \theta$ elliptical modes $\left(f_{B}=26.68 \mathrm{kHz}, f_{A}=26.75 \mathrm{kHz}, \Delta f=75 \mathrm{~Hz}\right.$, $Q_{A}=12.1 \mathrm{~K}, Q_{B}=14.8 \mathrm{~K}, \Delta Q=2700$, Vacuum: $30 \mu$ Torr).

mode-matched $(\Delta f=0)$. The tuning plots from two devices, one with low quadrature (device 1), and the other with high quadrature (device 2) are presented in Figure 7 and 8. Figure 7(a) shows the frequency change for the both $2 \theta$-modes for device 1 versus the tuning voltage applied to axis A. As shown in the Figure 7(b), the two frequencies can be tuned to a minimum $\Delta f=28 \mathrm{~Hz}$. The $2 \theta$ mode resonant frequency split ratio $(\Delta f / f)$ is minimized from $2.4 \%$ to $0.1 \%$ by applying a low $2.5 \mathrm{~V}$ tuning voltage in device 1 . Figure 8 (a) presents the tuning curves for device 2. With an initial $\Delta f$ of $550 \mathrm{~Hz}$ (Figure 8(b)), the minimum $\Delta f$ achieved is $290 \mathrm{~Hz}$, as shown in Figure $8(\mathrm{c})$. The $2 \theta$-mode resonant frequency split ratio $(\Delta f / f)$ is minimized from $2.2 \%$ to $1.1 \%$ by applying a $1.8 \mathrm{~V}$ tuning voltage in device 2. High quadrature error in this device makes modematching impossible.

In the hemispherical resonators studied here, the dominant quadrature error term results from the cross-axis stiffness component $k_{A B}$ (see Eqn. (3)). In other words, the resonator's primary axis of stiffness is not aligned with the sense electrodes. In addition, because the electrodes have a $45^{\circ}$ arc length, some A-axis vibration is always detected by the B-axis sensing electrodes (and vice-versa). A measure of this effect is the ratio of the capacitance change in the $\mathrm{A}$ and $\mathrm{B}$ axes resulting from $\mathrm{A}$-axis vibration, $\left(d C_{A} / d q_{A}\right) /\left(d C_{B} / d q_{A}\right)$. In an ideal gyro, this ratio would be infinite, since A-axis vibration $q_{A}$ would have no effect on the B-axis sense

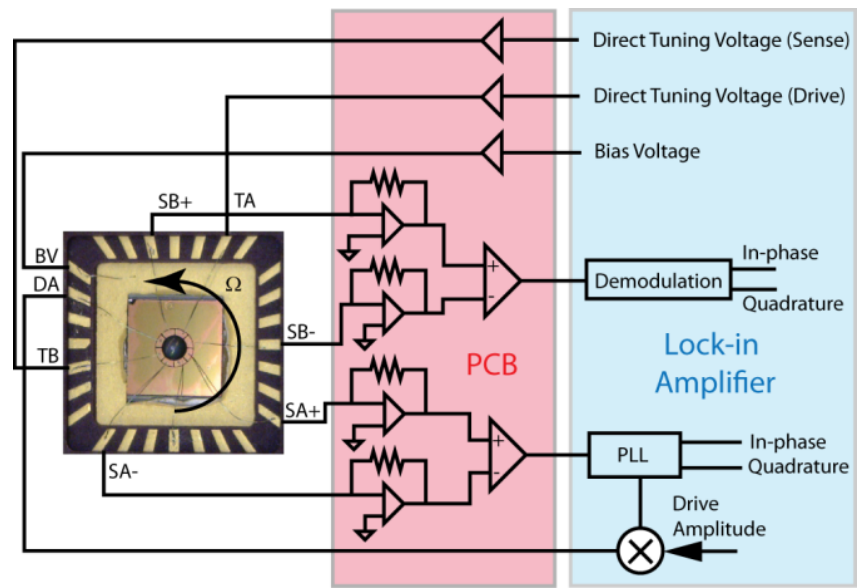

Figure 6: Testing platform used for gyroscope experiments. Key: tuning voltages $(T A, T B)$, drive voltages $(D A, D B)$, sensing voltages $(S A, S B)$, resonator bias voltage $(B V)$.
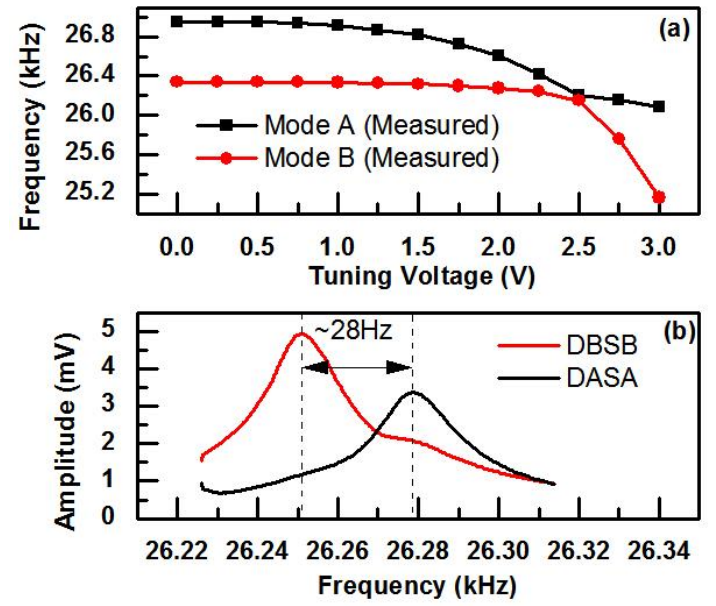

Figure 8: Device 1 results showing: (a) Mode A and mode B frequencies versus axis A tuning voltage; (b) Frequency responses from drive $A$ to sense $A$ (DASA) and drive $B$ to sense $B(D B S B)$ in the $2.5 \mathrm{~V}$ mode-matched condition.

capacitance $C_{B}$. In a real gyro, this ratio diminishes as the electrode's arc length is increased from $0^{\circ}$ to $45^{\circ}$, as shown in Figure 9. The plot also shows that the ratio depends on the angle $\theta$ of the primary axis of stiffness relative to the electrode axes. When $\theta=22.5^{\circ}$, the primary axis of stiffness is exactly midway between the A and B axis electrodes, corresponding to the worst case for quadrature error. The plot demonstrates that while large electrodes achieve high sense capacitance, they result in larger quadrature error.

Gyroscope experiments were conducted using a rate table (Aerosmith 1291BR). Rate table testing was conducted with a poor vacuum and as a result the observed $Q$ factors were over an order of magnitude lower than the values measured in vacuum probe station experiments. The sensitivity plots for two devices are shown in Figure 10 . Device 1 with lower $\Delta f / f$ of $0.1 \%$ and higher Q-factor of 1000, shows an order of magnitude greater scale factor than device 2 with higher $\Delta f / f$ of $1.2 \%$ and lower Q-factor of 600 . Device 1 achieved a scale factor of $140 \mu \mathrm{V} / \mathrm{deg} / \mathrm{s}$ while device 2 achieved a scale factor of $13 \mu \mathrm{V} / \mathrm{deg} / \mathrm{s}$. The difference in scale factor is due to the difference in $\mathrm{Q}$ and because device 2 was operated relatively far from the mode-matched condition.
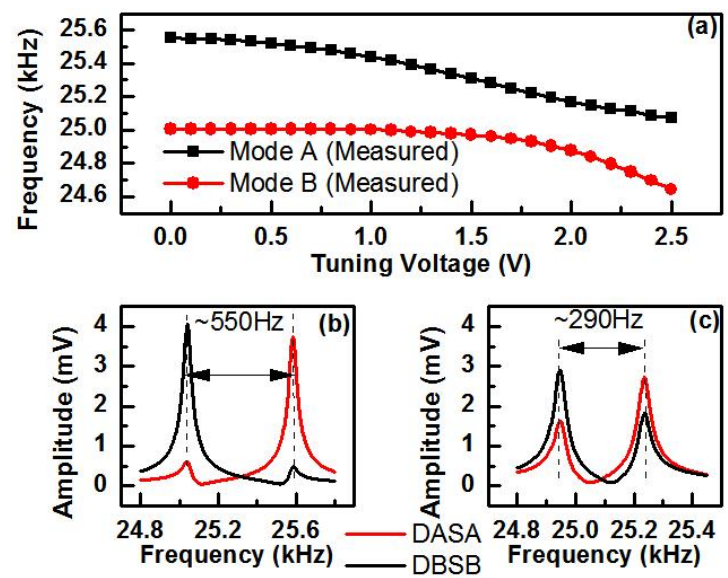

Figure 7: Device 2 results showing: (a) Mode A and mode B frequencies versus axis A tuning voltage; (b) As-fabricated frequency responses before mode-matching; (c) Frequency responses with the minimum achievable $\Delta f=290 \mathrm{~Hz}$ at $1.8 \mathrm{~V}$ tuning voltage. 


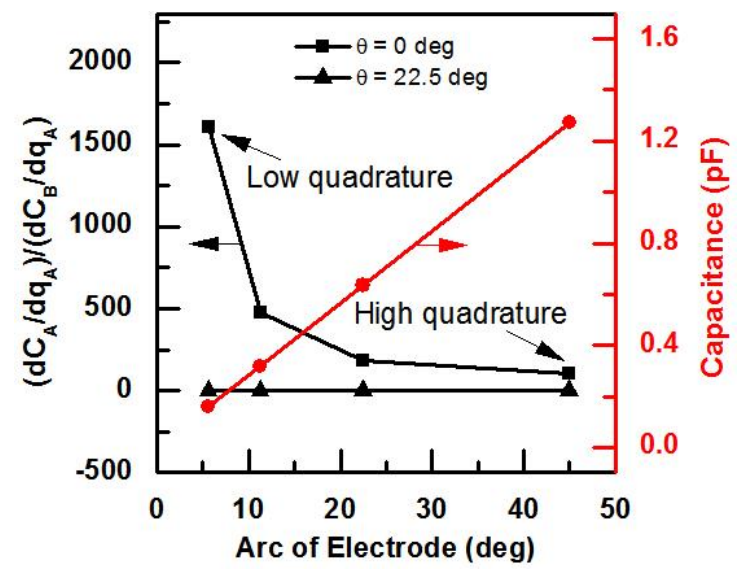

Figure 9: The ratio of the capacitance sensitivity to mode $A$ motion $\left(d C_{A} / d q_{A}\right) /\left(d C_{B} / d q_{A}\right)$ illustrates that mode $A$ vibration, $q_{A}$ couples into the mode $B$ sense capacitor, $C_{B}$ resulting in quadrature error. With quadrature nulling electrodes, the rotation of the primary axis of stiffness $(\theta)$ can be reduced from a maximum value of $22.5^{\circ}$ to $0^{\circ}$. In either case, large electrodes $\left(>15^{\circ}\right)$ result in high quadrature error.

The zero-rate output spectrum from both devices shows that the output noise is shaped by the gyro's mechanical frequency response, which has a $3 \mathrm{~dB}$ bandwidth of $27 \mathrm{~Hz}$ and $43 \mathrm{~Hz}$ for the two devices, respectively (Figure 11). Dividing the output noise at $0 \mathrm{~Hz}$ $(1.6 \mu \mathrm{V} / \sqrt{\mathrm{Hz}}$ and $1.1 \mu \mathrm{V} / \sqrt{\mathrm{Hz}})$ by the scale factor results in calculated angle random walk (ARW) of $11 \mathrm{mdeg} / \mathrm{s} / \sqrt{\mathrm{Hz}}$ and $85 \mathrm{mdeg} / \mathrm{s} / \sqrt{ } \mathrm{Hz}$, for devices 1 and 2 , respectively. The calculated ARW is approximately twice the theoretical Brownian noise $(700 \mathrm{nV} / \sqrt{\mathrm{Hz}})$ limit calculated due to electronic forcing noise contributed by the sense/drive electrodes. The electronic noise floor is $300 \mathrm{nV} / \sqrt{\mathrm{Hz}}$ for both devices.

\section{CONCLUSION}

In conclusion, we have reported the first micro-scale diamond hemispherical rate gyroscope. The gyroscope operates on the $2 \theta$ wineglass resonant mode using embedded electrodes with $1 \mu \mathrm{m}$ gap. The 8 electrode design is shown to allow mode-matching within $0.1 \%$ at $2.5 \mathrm{~V}$ tuning voltage in a device that has low initial quadrature error resulting in $140 \mu \mathrm{V} / \mathrm{deg} / \mathrm{s}$ scale factor and 11 mdeg/s/ $\sqrt{ } \mathrm{Hz}$ ARW.

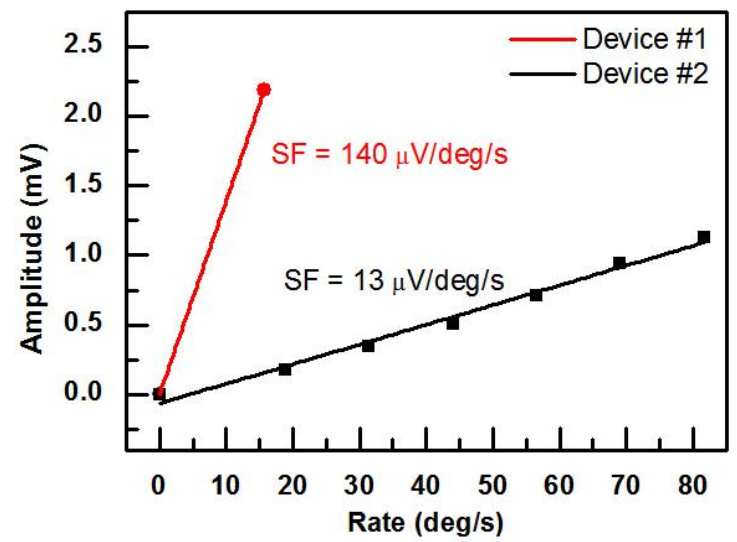

Figure 10: Rate table test results showing the output voltage versus input rate and the resulting scale factor $(S F)$.

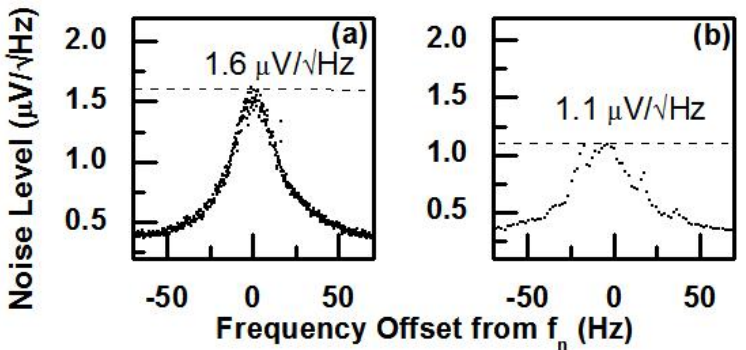

Figure 11: Zero rate output spectra for (a) device 1, (b) device

\section{ACKNOWLEDEGMENT}

This work is supported by DARPA under grant W31P4Q-111-0003. The authors would like to thank the support of the staff in UC Davis Northern California Nanotechnology Center and UC Berkeley Marvell Nanolab.

\section{REFERENCES}

[1] D. Senkal, M. J. Ahamed, A. A. Trusov, and A. M. Shkel, "Demonstration of Sub-1 Hz Structural Symmetry in MicroGlassblown Wineglass Resonators with Integrated Electrodes", Transducers 2013, pp. 1380-3.

[2] J. Cho, J. -K. Woo, J. Yan, R. L. Peterson, and K. Najafi, “A High-Q Birdbath Resonator Gyroscope (BRG)", Transducers 2013, pp. 1847-50

[3] L. D. Sorenson, X. Gao, and F. Ayazi, "3-D Micromachined Hemispherical Shell Resonators with Integrated Capacitive Transducers", MEMS 2012, pp. 168-71.

[4] A. Heidari, M.-L. Chan, H.-A. Yang, G. Jaramillo, P. TaheriTehrani, P. Fonda, H. Najar, K. Yamazaki, L. Lin, D. A Horsley, "Hemispherical wineglass resonators fabricated from the microcrystalline diamond", J. of Micromechanics and Microengineering, Vol. 23, No. 12, December 2013 , pp. 125016-23(8).

[5] B. J. Gallacher, J. Hedley, J. S. Burdess, A. J. Harris, A. Rickard, and D. O. King, "Electrostatic Correction of Structural Imperfections Present in a Microring Gyroscope", Journal of Microelectromechanical Systems, Vol. 14, No. 2, April 2005, pp. 221-34.

[6] J. Cho "High Performance Micromachined Vibratory Rate- and Rate-Integrating Gyroscopes" PhD dissertation, Electrical Engineering Department, The University of Michigan, 2012.

[7] S. Gao, J. Wu, "Theory and Finite Element Analysis of HRG", International Conference on Mechatronics and Automation, August 5-8, 2007, pp. 2768-72.

\section{CONTACT}

*Parsa Taheri-Tehrani; Tel: +1-530-341-3080;

ptaheri@ucdavis.edu 